\title{
Decaying dark matter and the tension in $\sigma_{8}$
}

\section{Kari Enqvist, ${ }^{a}$ Seshadri Nadathur, ${ }^{a}$ Toyokazu Sekiguchi ${ }^{a}$ and Tomo Takahashi $^{b}$}

\author{
${ }^{a}$ University of Helsinki and Helsinki Institute of Physics, P.O. Box 64, FI-00014, Helsinki, \\ Finland \\ ${ }^{d}$ Department of Physics, Saga University, Saga 840-8502, Japan \\ E-mail: kari.enqvist@helsinki.fi, toyokazu.sekiguchi@helsinki.fi, \\ seshadri.nadathur@helsinki.fi, tomot@cc.saga-u.ac.jp
}

\begin{abstract}
We consider decaying dark matter (DDM) as a resolution to the possible tension between cosmic microwave background (CMB) and weak lensing (WL) based determinations of the amplitude of matter fluctuations, $\sigma_{8}$. We perform N-body simulations in a model where dark matter decays into dark radiation and develop an accurate fitting formula for the non-linear matter power spectrum, which enables us to test the DDM model by the combined measurements of CMB, WL and the baryon acoustic oscillation (BAO). We employ a Markov chain Monte Carlo analysis to examine the overlap of posterior distributions of the cosmological parameters, comparing CMB alone with WL+BAO. We find an overlap that is significantly larger in the DDM model than in the standard CDM model. This may be hinting at DDM, although current data is not constraining enough to unambiguously favour a non-zero dark matter decay rate $\Gamma$. From the combined CMB+WL data, we obtain a lower bound $\Gamma^{-1} \geq 97$ Gyr at $95 \%$ C.L, which is less tight than the constraint from CMB alone.
\end{abstract}




\section{Contents}

1 Introduction 1

2 Model and linear perturbation calculation $\quad 3$

2.1 Model of decaying dark matter 3

2.2 Background evolution 3

2.3 Linear perturbation evolution and matter power spectrum 4

3 N-body simulation and non-linear matter power spectrum $\quad 6$

3.1 Simulation 6

$\begin{array}{lll}3.2 & \text { Non-linear matter power spectrum } & 6\end{array}$

4 Constraints from CMB and weak lensing measurements $\quad 8$

5 Conclusion $\quad 11$

$\begin{array}{ll}\text { A Approximations in solving Boltzmann hierarchy } & 12\end{array}$

B Fitting formula for non-linear matter power spectrum in DDM model 14

\section{Introduction}

Observations of temperature and polarization anisotropies in the cosmic microwave background $(\mathrm{CMB})$ have been a milestone in cosmology, which allow precision tests of various cosmological models. The six-parameter $\Lambda$ Cold Dark Matter $(\Lambda \mathrm{CDM})$ model has emerged as the concordance model of the Universe due to its ability to fit the CMB anisotropies and various other cosmological observations. ${ }^{1}$ The recent observation of CMB by Planck allows us to determine the cosmological parameters in this model to sub-percent accuracy [1].

At the same time, closer analysis of the level of consistency of the CMB and other cosmological data has revealed a number of possible tensions in the $\Lambda \mathrm{CDM}$ model. One that has recently received attention is the value of the amplitude of matter fluctuations, characterized by the parameter $\sigma_{8}$. The value of $\sigma_{8}$ estimated from observations of largescale structure such as the weak lensing of distant galaxies and abundance of galaxy clusters is significantly smaller than the one inferred from the Planck CMB power spectrum $[2-5] .{ }^{2}$ This tension might be indicating a need to move beyond the standard $\Lambda \mathrm{CDM}$ and to search for a mechanism that would suppress the matter fluctuations at late times.

Several authors have argued that the tension can be alleviated if active neutrinos are massive or sterile neutrinos exist $[3,4,6-8] .{ }^{3}$ However, this is not the only possible solution for the tension, and other models should also be tested on equal footing. A better understanding of effects of astrophysical processes might also be important (see, e.g., Ref. [10]). In this paper

\footnotetext{
${ }^{1}$ See Ref. [1] and references therein.

${ }^{2}$ Most of these studies were based on the Planck 2013 results, but the discrepancy still exists in the recent Planck 2015 data [1].

3 The suppression of $\sigma_{8}$ has also been discussed recently in a specific model that combines dark matter and dark radiation [9].
} 
we explore another possibility, where dark matter can decay with a lifetime $\Gamma^{-1}$ of the order of the age of the Universe.

Such a decaying dark matter (DDM) model is viable if the decay products interact little with the visible particles; otherwise the lifetime should be much longer. ${ }^{4}$ In this paper, we restrict ourselves to the simplest class of DDM models, where decay products are invisible and massless. In addition, we also assume that all the dark matter consists of DDM. Such a model can be characterised with only one extra parameter $\Gamma$ in addition to the standard six parameters of the $\Lambda \mathrm{CDM}$ model. While one can consider more complicated models of DDM with, e.g., finite masses of decay products or a mixture of DDM and CDM, our analysis of the simplest case is sufficient to demonstrate the potential of DDM models.

In DDM models, the formation of cosmic structure is in general suppressed. This is because the decay products free-stream with finite kick-velocity and can escape from the gravitational potential wells surrounding matter over-densities. On the other hand, at early times before significant decay has occurred, DDM models are completely degenerate with the CDM case. This allows the amplitude of matter fluctuations at late time to be suppressed relative to the CDM case, without significantly affecting the CMB anisotropy spectrum except through the integrated Sachs-Wolfe effect.

To distinguish DDM from CDM using cosmological observations requires a precise knowledge of the matter power spectrum over a wide range of scales. On large scales, structure formation in DDM models can be described by linear perturbation theory and has been studied by many authors [12-18]. Based on this linear approach and using the Planck CMB power spectrum combined with measurements of baryon acoustic oscillations (BAO) and the galaxy power spectrum on linear scales, Ref. [18] gives a lower bound $\Gamma^{-1} \geq 160 \mathrm{Gyr}$ on the lifetime of DDM. ${ }^{5}$

On the other hand, to make use of other cosmological data requires capturing the behaviour of the matter power spectrum to sufficient accuracy on non-linear scales, which can be achieved through using N-body simulations. ${ }^{6}$ In this paper we modify the Gadget2 code $[23,24]$ to incorporate the effects of DDM.

N-body simulations of DDM models have recently been used to study the effects on halo inner profiles [25, 27], the Lyman- $\alpha$ forest [26] and effects on non-linear matter power spectrum [27]. The implications for small-scale problems in the CDM models have also been discussed in Ref. [29]. In these studies, decay products were assumed to be non-relativistic. On the other hand, we focus on models with massless decay product, and follows the methodology developed in Ref. [28]. We will show that our linear calculations and N-body simulations agree well on scales where non-linearity is subdominant. We develop a fitting formula which can describe non-linear matter power spectrum well, using which we are able to obtain parameter constraints in the DDM model from observational data, especially including the recent CFHTLens weak lensing measurement [30].

We note that the possibility for DDM to resolve the tension in the $\sigma_{8}$ estimations has also been discussed in Refs. [17, 27,31], although the models and parameter regions of DDM they consider differ from ours. Also, in this paper we focus on the weak lensing measurement, which has not been considered in previous DDM studies.

The structure of this paper is as follows. In Section 2, we define the DDM model and discuss the cosmological linear perturbation theory. In Section 3, we describe our N-body

\footnotetext{
${ }^{4}$ For a recent constraint, we refer to Ref. [11].

${ }^{5}$ Ref. [19] gives a comparable constraint, $\Gamma^{-1} \geq 100 \mathrm{Gyr}$, using only information on distances.

${ }^{6}$ For semi-analytic studies based on the halo model see, e.g., Refs [15, 20-22].
} 
simulations and discuss the effects of DDM on the non-linear matter power spectrum. Using the simulation results, we obtain constraints on the DDM model from current cosmological observations in Section 4. In particular, we examine to what extent the DDM model can mitigate the tension in the $\sigma_{8}$ estimations. We conclude in the Section 5. In Appendix A, we describe the approximations used in the Boltzmann hierarchy in the linear calculation. Appendix B contains details of our fitting formula for the non-linear matter power spectrum.

Throughout this paper, we denote a derivative with respect to the conformal time $\tau$ by a dot, i.e. $\equiv \partial / \partial \tau$. We will assume a flat Universe and adiabatic initial perturbations with a power-law primordial power spectrum.

\section{Model and linear perturbation calculation}

\subsection{Model of decaying dark matter}

We consider a model of dark matter (DM) decaying into dark radiation (DR) with decay rate $\Gamma$. We assume the DM is non-relativistic. Note that as long as the decay products are massless, the number of DR particles generated from decay of single DM is irrelevant to the evolution of the cosmological perturbations.

Roughly speaking, current observations constrain the lifetime $\Gamma^{-1}$ to be larger than $100 \mathrm{Gyr}[18,19]$, so we focus on values of $\Gamma^{-1}$ around 100 Gyr in obtaining observational constraints.

\subsection{Background evolution}

Given a model of DDM, energy conservation for the DM and DR fluids in the unperturbed background metric yields

$$
\begin{aligned}
\dot{\bar{\rho}}_{d m}+3 \mathcal{H} \bar{\rho}_{d m} & =-a \Gamma \bar{\rho}_{d m} \\
\dot{\bar{\rho}}_{d r}+4 \mathcal{H} \bar{\rho}_{d r} & =a \Gamma \bar{\rho}_{d m},
\end{aligned}
$$

where $\bar{\rho}_{i}$ is the unperturbed energy density of fluid $i$ and $\mathcal{H} \equiv \dot{a} / a$ is the conformal Hubble parameter.

Due to the energy transfer between DM and DR fluids, $\bar{\rho}_{d m}$ and $\bar{\rho}_{d r}$ cannot be represented as explicit functions of $a$. This means that the present value of the Hubble parameter, $H_{0}=100 \mathrm{~h} \mathrm{~km} / \mathrm{sec} / \mathrm{Mpc}$, cannot be given as an input parameter but is rather a derived parameter obtained by solving the differential equations (2.1) and (2.2) in conjunction with the Friedmann equation

$$
\mathcal{H}^{2}=8 \pi G a^{2} \sum_{i} \bar{\rho}_{i} / 3
$$

where $G$ is Newton's constant.

To specify the background evolution, it is convenient to introduce the following quantity:

$$
\tilde{\omega}_{i}(a)=\frac{\bar{\rho}_{i}(a) a^{3\left(1+w_{i}\right)}}{\rho_{\text {crit }} / h^{2}}
$$

where $\rho_{\text {crit }} / h^{2} \equiv 3(100 \mathrm{~km} / \mathrm{sec} / \mathrm{Mpc})^{2} / 8 \pi G$ and $w_{i}$ is the (constant) equation of state parameter of fluid $i$. Given the initial values $\tilde{\omega}_{i}(a=0) \equiv \omega_{i}$, we can solve the differential equations (2.1) and (2.2) for $\tilde{\omega}_{d m}$ and $\tilde{\omega}_{d r}$ to obtain $\Omega_{i} h^{2} \equiv \tilde{\omega}_{i}(a=1)$ and $h$. Note that if fluid $i$ 
does not interact with other fluids (i.e., if the r.h.s. in Eqs. (2.1) and (2.2) vanish), $\tilde{\omega}_{i}(a)$ is constant in time and $\omega_{i}=\Omega_{i} h^{2}$.

The background cosmology is specified by the parameters

$$
\left(\omega_{b}, \omega_{d m}, \omega_{\Lambda}, \Gamma\right),
$$

where the subscripts $b$ and $\Lambda$ indicate respectively baryons and dark energy. We assume that initially $\omega_{d r}=0$ so that DR is produced only through the decay of DM. Given these parameters, $\Omega_{d m} h^{2}, \Omega_{d r} h^{2}$ and $h=\sqrt{\sum_{i} \Omega_{i} h^{2}}$ can be obtained as derived parameters. For later convenience, we define the reduced Hubble constant in the limit of no decay $\Gamma=0$, to be $h_{\emptyset}=\sqrt{\sum_{i} \omega_{i}}$. In this section and in Section 3, we adopt a fiducial set of initial density parameters:

$$
\left(\omega_{b}=0.02216, \omega_{d m}=0.119, \omega_{\Lambda}=0.318\right),
$$

and assume an amplitude $A_{s}=2.43 \times 10^{-9}$ and spectral index $n_{s}=0.963$ at a pivot scale $k=0.05 \mathrm{Mpc}^{-1}$ for the primordial power spectrum. This parameter set gives $h_{\emptyset}=0.677$ and corresponds to the best-fit $\Lambda \mathrm{CDM}$ model from the Planck CMB temperature power spectrum combined with measurements of small-scale CMB power spectrum and BAO [2].

In closing this section, in order to avoid confusion, let us clarify the difference between $\omega_{d m}$ and $\Omega_{d m} h^{2}$. As we noted, the density parameter $\tilde{\omega}_{d m}(t)$ is not constant and evolves with the time due to the decay of DM. We have defined the initial and final values of $\tilde{\omega}_{d m}$ as $\omega_{d m} \equiv \tilde{\omega}_{d m}(a=0)$ and $\Omega_{d m} h^{2} \equiv \tilde{\omega}(a=1)$. In the limit of no decay $\Gamma=0, \omega_{d m}$ and $\Omega_{d m} h^{2}$ coincide. Let us also remind the readers of the definitions of $h_{\emptyset} \equiv \sqrt{\sum_{i} \omega_{i}}$ and $h \equiv \sqrt{\sum_{i} \Omega_{i} h^{2}}$. The former is the reduced hubble parameter that are realized in the limit of no decay, while the latter is the actual reduced hubble parameter.

\subsection{Linear perturbation evolution and matter power spectrum}

Let us now discuss cosmological perturbation at linear order. We follow the notation of Ref. [32] and treat the perturbed quantities in the synchronous gauge of DM. In this gauge, the scalar part of the perturbed metric is

$$
d s^{2}=a(\tau)^{2}\left[d \tau^{2}+\left\{\delta_{i j}+h_{i j}(\vec{x}, \tau)\right\} d x^{i} d x^{j}\right],
$$

where $h_{i j}$ is given in Fourier space as

$$
h_{i j}(\vec{k}, \tau)=h_{\mathrm{L}}(\vec{k}, \tau) \hat{k}_{i} \hat{k}_{j}+6 \eta_{\mathrm{T}}(\vec{k}, \tau)\left(\hat{k}_{i} \hat{k}_{j}-\frac{1}{3} \delta_{i j}\right),
$$

and ^denotes a unit vector, i.e. $\hat{k}=\vec{k} / k$. In the following, we focus on a single Fourier mode and dependences on $\vec{k}$ will be abbreviated.

In this gauge, the continuity equation for DM is

$$
\dot{\delta}_{d m}=-\frac{\dot{h}_{\mathrm{L}}}{2},
$$

where $\delta_{d m}=\delta \rho_{d m} / \bar{\rho}_{d m}$ is the fractional overdensity of DM. Note that Eq. (2.9) is the same as in CDM.

On the other hand, since DR is massless, its perturbation equations can be given in terms of its brightness function defined as

$$
X(\hat{n}, \tau) \equiv \frac{1}{4 a^{4} \bar{\rho}_{d r}} \int \frac{q^{2} d q}{2 \pi^{2}} q \delta f_{d r}(\vec{q}, \tau),
$$




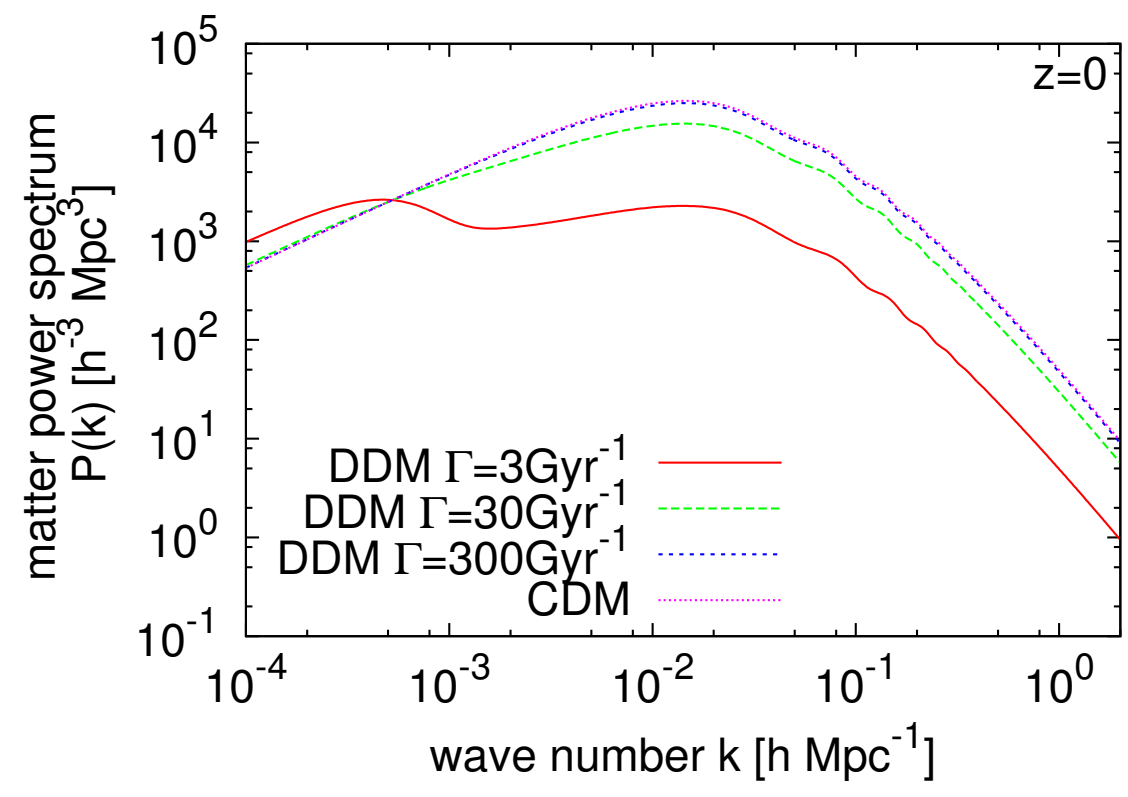

Figure 1. Power spectrum of non-relativistic matter today $(z=0)$, for DDM models with $\Gamma\left[\mathrm{Gyr}^{-1}\right]=3$ (red), 30 (green) and 300 (blue). For reference, the case with the standard CDM is also plotted (magenta).

where $\delta f_{d r}(\vec{q}, a)$ is the perturbation in the phase-space distribution of DR and $\hat{n}$ is the unit vector of a comoving momentum $\vec{q}=q \hat{n}$. The Boltzmann equation for DR can be given in terms of $X(\hat{n}, \tau)$ as [14]

$$
\dot{X}+i k \mu X=\left\{\dot{\eta}_{T}-\frac{1}{2}\left(\dot{h}_{L}+6 \dot{\eta}_{T}\right) \mu^{2}\right\}-\frac{\dot{\tilde{\omega}}_{d r}}{\tilde{\omega}_{d r}}\left(X-\frac{\delta_{d m}}{4}\right),
$$

where $\mu=\hat{k} \cdot \hat{n}$. After multipole expansion, Eq. (2.11) is recast into

$$
\dot{X}_{l}=\frac{k}{2 l+1}\left\{l X_{l-1}-(l+1) X_{l+1}\right\}-\frac{\dot{h}_{L}}{6} \delta_{l 0}+\frac{\dot{h}_{L}+6 \dot{\eta}_{T}}{15} \delta_{l 2}-\frac{\dot{\tilde{\omega}}_{d r}}{\tilde{\omega}_{d r}}\left(X_{l}-\frac{\delta_{d m}}{4} \delta_{l 0}\right),
$$

where $X(\hat{n}, \tau)=\sum_{l=0}^{\infty}(-i)^{l}(2 l+1) X_{l}(\tau) P_{l}(\mu)$, with $P_{l}$ being the Legendre polynomial.

To solve the perturbation equations, we have modified the Boltzmann code CAMB [33]. We truncated the Boltzmann hierarchy at $\ell_{\max }=50$ up to moderately sub-horizon scales, $k \tau \leq \ell_{\max }$. However, there is a difficulty in solving the Boltzmann hierarchy Eq. (2.12) on deep sub-horizon scales. A naïve truncation of the hierarchy yields fictitious reflections at $l_{\max }$, and the accumulation of them quickly blows up $X_{l}$. To avoid this, we adopted the approximations developed in Ref. [34], details of which are provided in Appendix A.

In Fig. 1, we plot the matter power spectrum $P(k)$ at present. It is clearly seen that as $\Gamma$ is increased, $P(k)$ is suppressed on small scales. We have also checked that our code reproduces the CMB power spectrum shown in Ref. [14] and the matter power spectrum in Ref. [18]. By adjusting the accuracy settings in CAMB, we have determined that within the parameter range of interest the numerical accuracy of the linear calculation code is sub- 
percent for the CMB temperature and polarization power spectra, and $\sim 1 \%$ for the matter power spectrum.

\section{$3 \quad$ N-body simulation and non-linear matter power spectrum}

\subsection{Simulation}

We perform N-body simulations of collisionless particles by using the public code Gadget2 [23, 24]. To incorporate effects of DDM, we make two modifications, which are also implemented previously in Ref. [28]. One is that the mass of N-body particles $m$ is made time-dependent and varies as

$$
m(t)=m_{i}\left\{\left(1-r_{d m}\right)+r_{d m} e^{-\Gamma t}\right\},
$$

where $m_{i}$ is the initial particle mass and $r_{d m} \equiv \omega_{d m} /\left(\omega_{b}+\omega_{d m}\right)$ is the initial fraction of the non-relativistic matter in DDM. The other is that the expansion of the Universe is given by solving Eqs. (2.1) and (2.2).

In this treatment, effects of perturbations in DR are neglected. However, since DR is relativistic and free-streams, on sub-horizon scales - where Newtonian gravity and thus the N-body simulation are valid - DR does not cluster and is highly homogeneous and isotropic. On the other hand, on super-horizon scales, the perturbations in DR cannot be neglected. However, on these scales, perturbations are linear and their evolution can be accurately computed within the perturbation theory treatment. Thus, by interpolating results of Nbody simulations on small scales and linear perturbation theory on large scales, we can predict the matter power spectrum in the DDM model over a broad range of scales.

The initial matter power spectrum at redshift $z=49$ is computed using CAMB, from which initial distributions of N-body particles are generated using the 2LPTic code [35], which uses second-order Lagrangian perturbation theory. The simulations contain $1024^{3}$ particles, and are performed with three different box sizes, $L=1250,500$ and $200 h_{\emptyset}^{-1} \mathrm{Mpc}$. For each box size, we generate three different realizations, and for each realization of the initial conditions, we run simulations with different values of $\Gamma$, which allows us to extract effects of the dark matter decay with statistical fluctuations minimised.

\subsection{Non-linear matter power spectrum}

Fig. 2 shows the ratio of the matter power spectrum in the DDM model with the lifetimes $\Gamma^{-1}=31.6,100$ and $316 \mathrm{Gyr}$ to that in the CDM model at redshifts $z=0$ and 1 . The power spectrum of N-body simulations is computed using the ComputePK code $[38]^{7}$ with the count-in-cell scheme, and the linear perturbation calculations are performed by using a modified version of the CAMB code as described in Section 2.3. Note that here we only take into account the overdensity of non-relativistic matter (i.e., baryons and DM), so DR is not included in computing the fractional overdensity. We also note that the units of horizontal axis are $\mathrm{Mpc}^{-1}$, which is different from the ordinary convention of $h \mathrm{Mpc}^{-1}$. This allows us to extract the difference in growth of density fluctuation, after removing the effects of the background expansion.

From Fig. 2, we can see that the ratio $P^{(\mathrm{DDM})}(k) / P^{(\mathrm{CDM})}(k)$ from the linear calculation and that from the N-body simulations coincide in the range $k \simeq 0.01-0.1 \mathrm{Mpc}^{-1}$, where the both the linear and Newtonian approximations should be valid. Since the effects of

\footnotetext{
${ }^{7}$ http://aramis.obspm.fr/ ${ }^{\sim}$ lhuillier/codes.php
} 


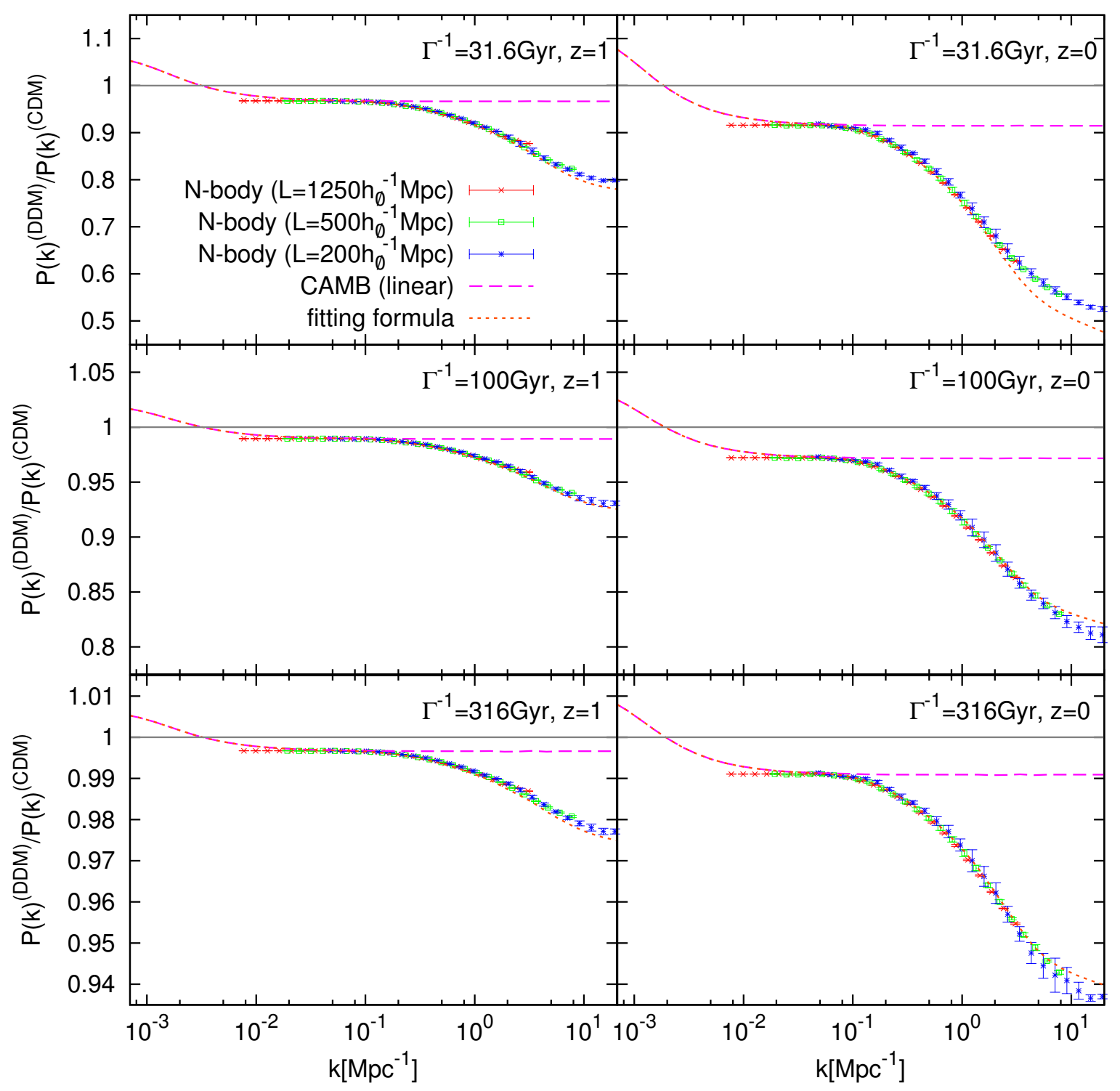

Figure 2. Ratio of non-linear matter power spectrum in the DDM model to that in the CDM model. Red, green and blue points with error bars are obtained from N-body simulations with box sizes $L=1250,500$ and $200 h_{\emptyset}^{-1} \mathrm{Mpc}$, respectively. The dashed magenta line is obtained from the linear perturbation calculation in the synchronous gauge by using CAMB. The dotted orange line is obtained by applying our fitting formula in Appendix B, which exactly overlaps with magenta line on large scales. The solid grey horizontal line indicates unity.

perturbations in the massless decay products become less relevant at smaller scales, this agreement validates our treatment of the decay products in the N-body simulations. 
The ratio $P^{(\mathrm{DDM})} / P^{(\mathrm{CDM})}$ in the linear calculation tends to increase as $k$ decreases below $10^{-2} \mathrm{Mpc}^{-1}$. This is because the decay product does not free-stream until perturbation scales cross the horizon, and its density perturbation more or less traces that of other nonrelativistic matter (see, e.g., Ref. [17] for detailed discussion). This effect is omitted in the N-body simulations, which results in no increase in the ratio on large scales.

As the scales become smaller, the non-linear matter power spectrum shows a larger deviation of the DDM model from the CDM case, while for the linear calculation the ratio stays almost constant. This is because the non-linear matter power spectrum is a non-linear function of linear power spectrum (with mode-mode couplings) and hence small deviations in linear perturbations are in general enhanced in the non-linear power spectrum. As nonlinearity becomes more prominent on smaller scales, the deviation becomes more enhanced.

It is also apparent that, except for changes in the overall magnitude, the fractional deviation $P^{(\mathrm{DDM})} / P^{(\mathrm{CDM})}-1$ has almost the same scale-dependence for various values of $\Gamma^{-1}$ and $z$. This allows us to obtain a fitting formula for the non-linear matter power spectrum in the DDM model in Appendix B, which is sufficiently accurate for a wide range of $\Gamma^{-1}$ and $z$. This fitting formula is shown by the orange lines in Fig. 2. Our formula can reproduce the fractional deviation $P^{(\mathrm{DDM})} / P^{(\mathrm{CDM})}-1$ from the N-body results to within an accuracy of $10 \%$.

One caveat is that our fitting formula is not tested against cosmological parameters other than the fiducial set described above. However, as shown in Fig. 5 in Appendix B, the ratio $\epsilon_{\text {non-linear }} / \epsilon_{\text {linear }}(k)$, where $\epsilon(k)=1-P^{(\mathrm{DDM})} / P^{(\mathrm{CDM})}$, does not vary much with redshift or $\Gamma$. This suggests that our fitting formula will also depend only weakly on cosmological parameters. In addition, for the cosmic shear power spectrum, which measures the gravitational potential but not the fractional overdensity of matter, the impact of uncertainties in the fitting formula would further decrease. This is because deviations of the DDM model from the CDM case also arise from the decrease in mean energy density, which suppresses the shear power spectra additionally by $\simeq\left\{\left(1-r_{d m}\right)+r_{d m} e^{-\Gamma t}\right\}^{2} \simeq 1-2 r_{d m} \Gamma t$. The suppression from this effect is in general larger than the contribution from the power spectrum of fractional matter overdensity. Thus the weak dependence of our fitting formula on cosmological parameters should not significantly affect the following analysis.

\section{Constraints from CMB and weak lensing measurements}

Having obtained a fitting formula for the non-linear matter power spectrum in the DDM model, we now compare the model predictions to the data on weak lensing, CMB and BAO, using a modified CosmoMC code [36]. To obtain the non-linear power spectrum in the DDM model, we combine our fitting formula for the $P^{(\mathrm{DDM})} / P^{(\mathrm{CDM})}$ ratio with the improved HALOFIT formula of Ref. [45].

For the CMB data, we use the Planck 2013 temperature maps [39] combined with the WMAP 9-year polarization data [40], and denote the combination as CMB. For weak lensing data, we use the tomographic analysis of the CFHTLens data by Ref. [30], which we denote as WL. In particular, we adopt the tomographic cosmic shear power spectrum from blue galaxy samples with $N_{t}=6$ redshift bins and the "conservative" cut on small angles to mitigate systematic errors from baryonic effects. We refer the reader to Ref. [30] for more details. We also use the measurements of baryon acoustic scales from galaxy surveys [41-43] and the Planck CMB lensing power spectrum [44], which are denoted as BAO and lensing, respectively. We use four different combinations of these data, which are WL+BAO, CMB 
alone, $\mathrm{CMB}+\mathrm{WL}$, and $\mathrm{CMB}+\mathrm{WL}+\mathrm{BAO}+$ lensing. For the $\mathrm{WL}+\mathrm{BAO}$ combination, we impose Gaussian priors $\omega_{b}=0.0223 \pm 0.0009$ and $n_{s}=0.96 \pm 0.02$. Here the ranges for the Gaussian priors are taken to be somewhat broader than the $1 \sigma$ errors in the Planck measurements of these quantities. However, the prior range does not affect our final results much since $\mathrm{WL}$ and BAO are not sensitive to $\omega_{b}$ and $n_{s}$. (In fact, even if we fix the values of $\omega_{b}$ and $n_{s}$ in obtaining the constraints from $\mathrm{WL}+\mathrm{BAO}$, the results do not change much.)

The cosmic shear power spectrum is obtained as follows. Using the Limber approximation, the cross-angular power spectrum of the convergence fields $\kappa$ at redshifts $z_{1}$ and $z_{2}$ at multipole $\ell$ is given as (see, e.g., Ref. [37])

$$
C_{\ell}^{\kappa}\left(z_{1}, z_{2}\right)=\int d r\left(1-\frac{r}{r_{1}}\right)\left(1-\frac{r}{r_{2}}\right)\left[4 \pi G a^{2} \bar{\rho}(a)\right]^{2} P\left(k=\frac{\ell}{r}, z\right),
$$

where $r(z)$ is the comoving distance to redshift $z=1 / a-1$, and $r_{i}=r\left(z_{i}\right), i=1,2$. Note that we have omitted the contribution from perturbations of the decay products, because current observations are sensitive to perturbations at sub-horizon scales, where those of decay products can be neglected. By applying the fitting formula for the non-linear matter power spectrum in the DDM model, Eq. (4.1) can provide the theoretical prediction for weak lensing observations.

In order to avoid confusion, let us remind the reader of the definitions of parameters which we constrain in the following. $\omega_{d m}$ is the initial density parameter of dark matter and in general differs from one at present, $\Omega_{d m} h^{2} ; \sigma_{8}$ is computed from the matter power spectrum of non-relativistic matter; $H_{0}=100 h$ is the actual Hubble parameter at present and should not be confused with $100 h_{\emptyset}$. The definitions of these parameters are presented in Sec. 2.2.

Let us take a look at the parameter constraints in the CDM model first. Fig. 3 shows the constraints on parameters of particular interest, $\omega_{d m}, \sigma_{8}$ and $H_{0}$. In the $\omega_{d m}-\sigma_{8}$ and $H_{0}-\sigma_{8}$ planes, the posterior distributions from the $\mathrm{CMB}$ and $\mathrm{WL}+\mathrm{BAO}$ datasets overlap only marginally at the $95 \%$ C.L. level. In particular, the tension resides in estimation of $\sigma_{8}$. This result highlights the existence of the tension between Planck and CFHTLens, which has been argued in the literature $[1,2,5,7]$. To be more quantitative, we explore the posterior distributions from these two datasets in the 3-dimensional parameter space of $\left(\omega_{d m}, \sigma_{8}\right.$, $H_{0}$ ). We find that the posterior distributions overlap only at more than $90 \%$ C.L., which is broadly consistent with the results of Ref. [7], where the full 6-dimensional parameter space is analysed.

Fig. 4 shows parameter constraints in the DDM model, which has an extra parameter $\Gamma$. Compared to the CDM case, the WL $+\mathrm{BAO}$ dataset allows a larger value of $\sigma_{8}$. This leads to a substantial reduction of the tension between Planck and CFHTLens in the CDM model. In the same manner as in the CDM model, we examine the overlap of the posterior distributions from $\mathrm{CMB}$ and $\mathrm{WL}+\mathrm{BAO}$ in the 4-dimemsional parameter space $\left(\Gamma, \omega_{d m}, \sigma_{8}\right.$, $H_{0}$ ) of the DDM model, and find that they overlap at $55 \%$ C.L. This level of improvement appears somewhat better than that achieved by the addition of massive (sterile) neutrinos [7], which reports that posterior distributions from CFHTLens and CMB overlap at $90 \%$ C.L. in the massive neutrino model, that has one extra parameter, while they do so at $64 \%$ C.L. in the sterile neutrino model, that has two extra parameters. However, a direct comparison is complicated by the fact that the analysis uses the shear power spectrum data on smaller angular scales than we do, while additionally accounting for theoretical uncertainties due to intrinsic alignment of galaxies and the effects of AGN feedback. 


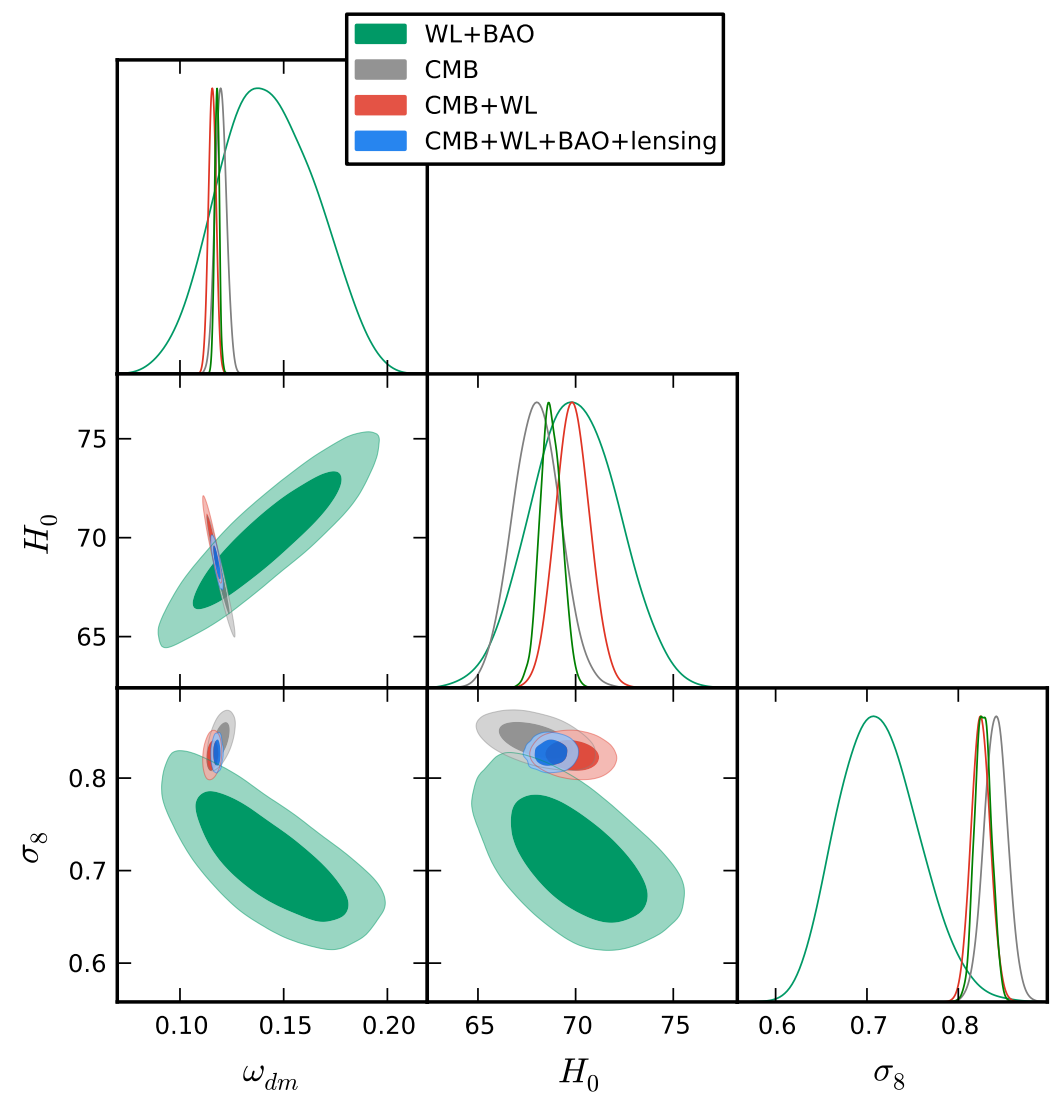

Figure 3. Constraints on the CDM model. The $1 \mathrm{~d}$ and $2 \mathrm{~d}$ posterior distributions for parameters $\omega_{d m}, H_{0}$ and $\sigma_{8}$ are shown, with the 2 d constraints given at $68 \%$ and $95 \%$ C.L. Green, grey, red and blue lines correspond to the data sets WL, CMB, CMB+WL and CMB+WL+BAO+lensing, respectively.

We would like to comment on the degeneracy between $\sigma_{8}$ and $\Gamma$ from the WL+BAO dataset. As seen from the bottom-left panel in Fig. 4, these two parameters are positively correlated, which may at first look counterintuitive as matter fluctuations should be more suppressed as $\Gamma$ becomes larger. However, $\sigma_{8}$ represents the amplitude of the overdensity in non-relativistic matter (baryon+DM), which is less suppressed than that in the total matter (i.e. baryon $+\mathrm{DM}+\mathrm{DR}$ ) or gravitational potential. Therefore, as $\Gamma$ is increased, $\sigma_{8}$ should be also increased to fit the amplitude of the cosmic shear power spectrum.

Finally, we present constraints on the lifetime of DDM, $\Gamma^{-1}$, from the various datasets. From the CMB alone, we obtain $\Gamma^{-1} \geq 140 \mathrm{Gyr}$ at $95 \%$ C.L. This is very similar to the constraints in Ref. [18], where the authors use CMB+BAO combined with the galaxy power spectrum. Combining CMB with WL, we obtain a less stringent constraint, $\Gamma^{-1} \geq 97$ Gyr. This is because the CMB+WL data prefers a nonzero value of $\Gamma$, in order to mitigate the $\sigma_{8}$ tension in the CDM model. Inclusion of $\mathrm{BAO}$ and the CMB lensing data changes the lower bound only by a few percent. On the other hand, WL data alone is not yet sufficiently constraining even when it is combined with $\mathrm{BAO}$, so we cannot obtain meaningful constraints on $\Gamma^{-1}$ above $\Gamma^{-1} \geq 31.6 \mathrm{Gyr}$, where we can guarantee the accuracy of our fitting formula. 


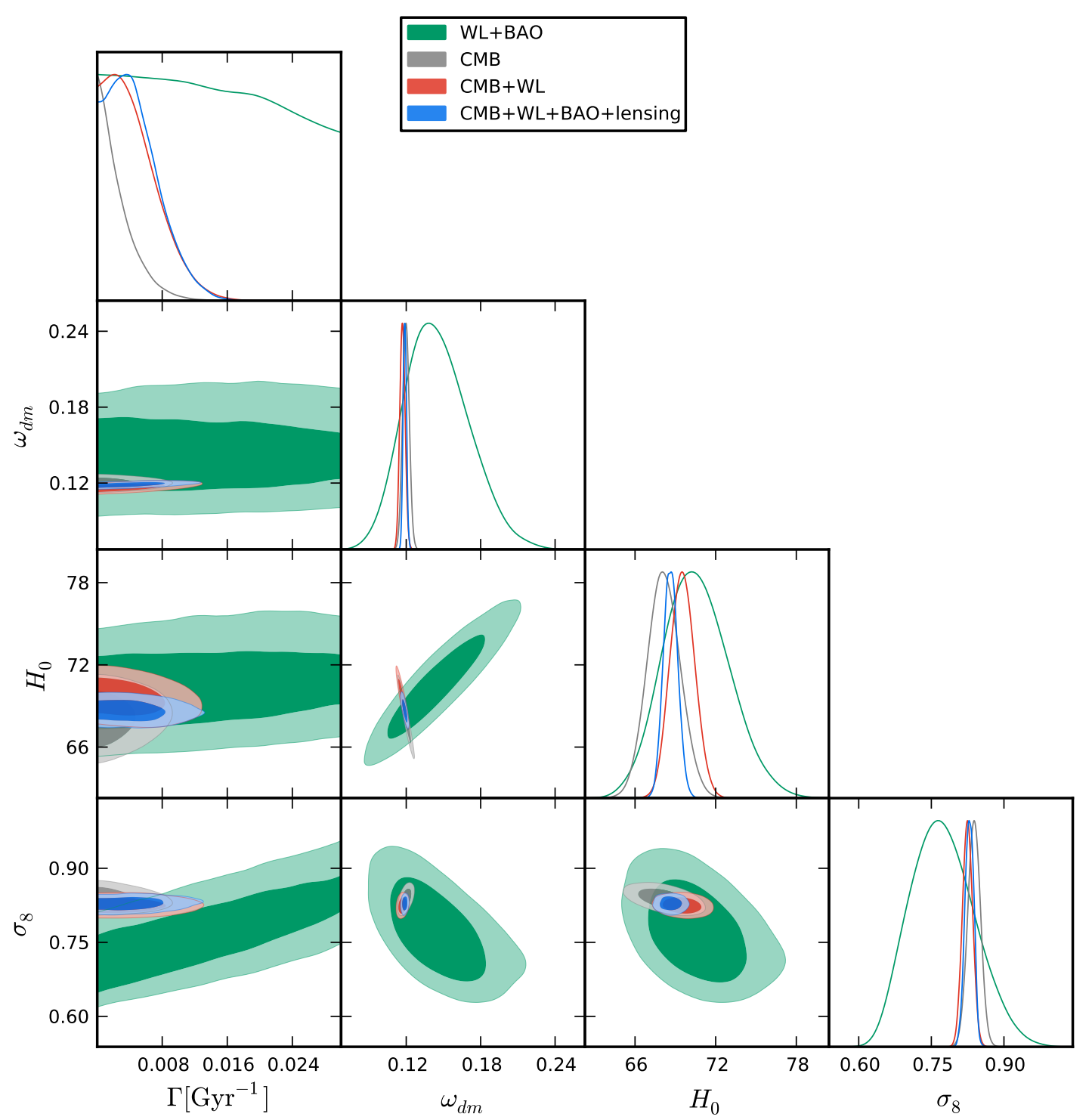

Figure 4. The same as in Fig. 3, but for the DDM model, which has an additional parameter $\Gamma$.

\section{Conclusion}

We have studied cosmological structure formation in a model of dark matter decaying into massless invisible particles ("dark radiation"), denoted as DDM model. Employing N-body simulations, we have obtained an accurate fitting formula for the non-linear matter power spectrum in the DDM model. Using this fitting formula, we compared predictions of DDM to the tomographic cosmic shear power spectrum measurements from the CFHTLens survey in conjunction with $\mathrm{CMB}$ and $\mathrm{BAO}$ data. We have demonstrated that the tension present in estimations of $\sigma_{8}$ in the CDM model is substantially alleviated in the DDM model.

Quantitatively, we find that the posterior distributions from two different datasets, CMB alone and WL+BAO, overlap with each other at $55 \%$ C.L. in the DDM model, while they 
do so only at $90 \%$ C.L. in the CDM model. We thus suggest the $\sigma_{8}$ discrepancy may be hinting at the possibility that dark matter could be unstable. On the other hand, current cosmological observations do not provide powerful enough constraints to nail down the finite lifetime of DM, $\Gamma^{-1}$. Combining the CFHTLens weak lensing data with the Planck and WMAP CMB data, we obtain a lower bound, $\Gamma \geq 97$ Gyr. This is less stringent than the one from CMB alone, $\Gamma \geq 140 \mathrm{Gyr}$, which reflects the preference for a non-zero dark matter decay width from $\mathrm{CMB}+\mathrm{WL}$.

In the near future weak lensing measurements such as the Subaru Hyper SuprimeCam [46], the Dark Energy Survey [47], and Euclid [48], should increase the statistics of the data by orders of magnitude. The DDM scenario we have considered in this paper will thus definitely be tested by these future observations. One should also point out that certain cosmological observations other than weak lensing may also show signs of tension with the $\mathrm{CMB}$ and the $\Lambda \mathrm{CDM}$ model. In particular, the number of galaxy clusters observed by the Sunyaev-Zeldovich effect and X-ray observations are known to be less than the prediction of the best-fit $\Lambda$ CDM model to the CMB power spectrum [2]. Since the amplitude of matter fluctuations are suppressed in the DDM model, the abundance of dark matter haloes and hence hosted galaxy clusters also becomes less than in the CDM model. It would be interesting to consider whether the DDM model can in a consistent manner resolve the possible disagreements between all such observations. We leave this discussion to a future work.

\section{Acknowledgments}

T.S. would like to thank Naoshi Sugiyama for valuable comments and suggestions. T.T. would like to thank the Helsinki Institute of Physics for the hospitality during the visit, where a part of this work has been done. The work of T.T. is partially supported by the Grant-in-Aid for Scientific research from the Ministry of Education, Science, Sports, and Culture, Japan, No. 23740195. We thank the CSC - IT Center for Science (Finland) for computational resources.

\section{A Approximations in solving Boltzmann hierarchy}

In this appendix, we summarise the approximations used in solving the Boltzmann hierarchy of Eq. (2.12) in the linear perturbation calculation. Our approximations are based on Ref. [34].

First of all, let us define a new variable $\tilde{X} \equiv \tilde{\omega}_{d r} X$. Then Eq. (2.11) can be rewritten as

$$
\dot{\tilde{X}}+i k \mu \tilde{X}=\tilde{\omega}_{d r}\left\{\dot{\eta}_{T}-\frac{1}{2}\left(\dot{h}_{L}+6 \dot{\eta}_{T}\right) \mu^{2}\right\}+\dot{\tilde{\omega}}_{d r} \frac{\delta_{d m}}{4} .
$$

Noting that $\tilde{X}_{l}$ vanishes at $\tau=0$, the formal solution of this equation can be given as

$$
\tilde{X}(\tau)=\int_{0}^{\tau} d \tau^{\prime} e^{-i k \mu\left(\tau-\tau^{\prime}\right)}\left\{A\left(\tau^{\prime}\right)+B\left(\tau^{\prime}\right) \mu^{2}\right\}
$$

where

$$
\begin{aligned}
& A=\tilde{\omega}_{d r} \dot{\eta}_{T}+\dot{\tilde{\omega}}_{d r} \frac{\delta_{d m}}{4}, \\
& B=-\frac{\tilde{\omega}_{d r}}{2}\left(\dot{h}_{L}+6 \dot{\eta}_{T}\right) .
\end{aligned}
$$


Since $B$ vanishes at $\tau=0$, after integrating by part and multipole expansion, we obtain

$$
\begin{aligned}
& \tilde{X}_{l}(\tau)-\frac{1}{k^{2}}\left(\dot{B}(\tau) \delta_{l 0}+\frac{k}{3} B(\tau) \delta_{l 1}\right) \\
& =-\frac{\dot{B}(0)}{k^{2}} j_{l}(k \tau)+\int_{0}^{\tau} d \tau^{\prime} j_{l}\left(k\left(\tau-\tau^{\prime}\right)\right)\left(A\left(\tau^{\prime}\right)-\frac{\ddot{B}\left(\tau^{\prime}\right)}{k^{2}}\right),
\end{aligned}
$$

where $j_{l}$ is the first kind of spherical Bessel function.

After horizon crossing $k \tau \simeq 1, \tilde{X}_{l}$ starts to oscillate as the free-streaming generates higher multipole moments from lower ones. In this regime, the ultra-relativistic fluid approximation (UFA) developed in Ref. [34] can be applied. Following Ref. [34], let us introduce a new variable $Y_{l}=\tilde{X}_{l}-\frac{1}{k^{2}}\left(\dot{B} \delta_{l 0}+\frac{k}{3} B \delta_{l 1}\right)$, whose behaviour is quite close to that of $j_{l}(k \tau)$, which satisfies $j_{l}^{\prime}(x)-j_{l-1}(x)+\frac{l+1}{x} j_{l}(x)=0$. From Eq. (A.5), one can derive the following equation:

$$
\begin{aligned}
\dot{Y}_{l} & -k Y_{l}+\frac{l+1}{\tau} Y_{l} \\
& =\left\{A(\tau)-\frac{\ddot{B}}{k^{2}}(\tau)\right\} \delta_{l 0}-\frac{l+1}{\tau} \int_{0}^{\tau} d \tau^{\prime} \frac{\tau^{\prime}}{\tau-\tau^{\prime}} j_{l}\left(k\left(\tau-\tau^{\prime}\right)\right)\left\{A\left(\tau^{\prime}\right)-\frac{\ddot{B}\left(\tau^{\prime}\right)}{k^{2}}\right\} .
\end{aligned}
$$

The integrand on the r.h.s becomes large only at $k \tau-l \lesssim k \tau^{\prime} \lesssim k \tau$. This allows one to approximate the third term on the r.h.s by

$$
-(l+1)\left\{A(\tau)-\frac{\ddot{B}(\tau)}{k^{2}}\right\} \int_{0}^{\infty} d x \frac{j_{l}(x)}{x} .
$$

In particular, for $l=2$, Eq. (A.6) can be approximately rewritten in terms of $X_{l}$ as

$$
\dot{X}_{2}=k X_{1}-\left(\frac{3}{\tau}+\frac{\dot{\tilde{\omega}}_{d r}}{\tilde{\omega}_{d r}}\right) X_{2}+\frac{\dot{h}_{L}}{6}+2 \dot{\eta}_{T}+\frac{\dot{\tilde{\omega}}_{d r}}{\tilde{\omega}_{d r}} \frac{\delta_{d m}}{4}
$$

where we have assumed $\left|\ddot{B} / k^{2}\right| \sim\left|B /(k \tau)^{2}\right| \ll|A|$.

On the other hand, deeper inside horizon, perturbations in $X_{l}$ become very small and their effects on the perturbation evolution of non-relativistic matter are not relevant unless the energy density of the decay product dominates the Universe. On such scales, the oscillation period of $X_{l}$ is much shorter than the typical time-scale of perturbation evolution of nonrelativistic matter, so that only non-oscillatory components of $X_{l}$ suffice in computing the matter perturbation evolution. In this regime, the radiation streaming approximation (RSA) in Ref. [34] can be applied. Let us consider the Boltzmann hierarchy for $\tilde{X}_{l}$ :

$$
\begin{aligned}
\dot{\tilde{X}}_{0} & =-k \tilde{X}_{1}-\tilde{\omega}_{d r} \frac{\dot{h}_{L}}{6}+\dot{\tilde{\omega}}_{d r} \frac{\delta_{d m}}{4}, \\
\dot{\tilde{X}}_{1} & =\frac{k}{3} \tilde{X}_{0}
\end{aligned}
$$

where we have assumed $\tilde{X}_{l}=0$ for $l \geq 2$. Combining these two equations we obtain

$$
X_{0}=\frac{1}{\tilde{\omega}_{d r} k^{2}} \frac{\partial}{\partial \tau}\left(-\tilde{\omega}_{d r} \frac{\dot{h}_{L}}{2}+\dot{\tilde{\omega}}_{d r} \frac{3 \delta_{d m}}{4}\right),
$$


where we have assumed $\left|\ddot{\tilde{X}}_{0}\right| \ll k^{2}\left|\tilde{X}_{0}\right|$ since we are interested in the non-oscillatory components of $\tilde{X}_{l}$. By integrating Eq. (A.9), we then obtain

$$
X_{1}=-\frac{\dot{h}_{L}}{6 k}+\frac{\dot{\tilde{\omega}}_{d r}}{\tilde{\omega}_{d r}} \frac{\delta_{d m}}{4 k},
$$

where we have used the fact that $\tilde{X}_{l}, \tilde{\omega}_{d r} \dot{h}_{L}$ and $\dot{\tilde{\omega}}_{d r} \delta_{d m}$ vanish at $\tau=0$. To compute $\ddot{h}_{L}$, we adopt the same procedure as in Ref. [34], which is valid in a matter-dominated Universe.

In our calculation, the Boltzmann hierarchy Eq. (2.12) truncated at $l=l_{\max }$ is directly solved from superhorizon scales up to $k \tau=l_{\max }$. Then we switch the UFA on and the Boltzmann hierarchy is truncated at $l=2$ by adopting Eq. (A.8). Finally, when $k \tau \geq 10 \times l_{\max }$ in the matter dominated Universe, we switch the RSA on and substitute Eqs. (A.11)-(A.12) in the source term of the Einstein equation. These approximations avoid the unwanted blowup of $X_{l}$ caused by the truncation of Boltzmann hierarchy. Note that to employ the RSA, we need to assume that the decay product is a minor component of the energy density of the Universe. This assumption is valid in the parameter region we explore in this paper.

\section{B Fitting formula for non-linear matter power spectrum in DDM model}

In this appendix we present the fitting formula for the non-linear power spectrum of nonrelativistic matter in the DDM model. First, let us present the fitting formula for the suppression in the linear power spectrum $P_{\text {linear }}$ at the small scale limit. We find that $\epsilon_{\text {linear }}=1-P_{\text {linear }}^{(\mathrm{DDM})} / P_{\text {linear }}^{(\mathrm{CDM})}$ at $k \rightarrow \infty$ can be approximately given as

$$
\epsilon_{\text {linear }}(\Gamma, z)=\alpha\left(\frac{\Gamma}{\mathrm{Gyr}^{-1}}\right)^{\beta}\left(\frac{1}{z+1}\right)^{\gamma},
$$

where $\alpha, \beta$ and $\gamma$ are functions of $\omega_{b}, h_{\emptyset}$ and $\omega_{m}=\omega_{b}+\omega_{d m}$ in the case of our model, which can be fitted by

$$
\begin{aligned}
& \alpha\left(\omega_{b}, h_{\emptyset}, \omega_{m}\right)=(5.323-1.4644 u-1.391 v)+(-2.055+1.329 u+0.8672 v) w \\
&+(0.2682-0.3509 u) w^{2}, \\
& \beta\left(\omega_{b}, h_{\emptyset}, \omega_{m}\right)= 0.9260+(0.05735-0.02690 v) w+(-0.01373+0.006713 v) w^{2}, \\
& \gamma\left(\omega_{b}, h_{\emptyset}, \omega_{m}\right)=(1.653-0.7860 v)+(0.4884+0.1754 v) w \\
&+ \\
&+0.2512+0.07558 v) w^{2},
\end{aligned}
$$

where $u=\omega_{b} / 0.02216, v=h_{\emptyset} / 0.6777$ and $w=\omega_{m} / 0.1412$. The accuracy is $10 \%$ for $\Gamma^{-1} \geq 31$ Gyr and $z \leq 1$ as long as $0.019 \leq \omega_{b} \leq 0.026,0.6 \leq h_{\emptyset} \leq 0.8$ and $0.09 \leq \omega_{m} \leq 0.28$.

In Fig. 5 we show the suppression in the non-linear power spectrum $\epsilon_{\text {non-linear }}(k)=$ $1-P_{\text {non-linear }}^{(\mathrm{CDM})} / P_{\text {non-linear }}^{(\mathrm{DDM})}$ in units of $\epsilon_{\text {linear }}$ for $\Gamma^{-1}=31,100,316 \mathrm{Gyr}$ and $z=0,1$. The cosmological parameters in Eq. (2.6) are adopted here. One can see that $\epsilon_{\text {non-linear }}(k) / \epsilon_{\text {linear }}$ has only a weak dependence both on $\Gamma^{-1}$ and $z$. We find $\epsilon_{\text {non-linear }}(k) / \epsilon_{\text {linear }}$ can be fitted by the following functional form:

$$
\frac{\epsilon_{\text {non-linear }}(k)}{\epsilon_{\text {linear }}}=\frac{1+a\left(k / \mathrm{Mpc}^{-1}\right)^{p}}{1+b\left(k / \mathrm{Mpc}^{-1}\right)^{q}}
$$




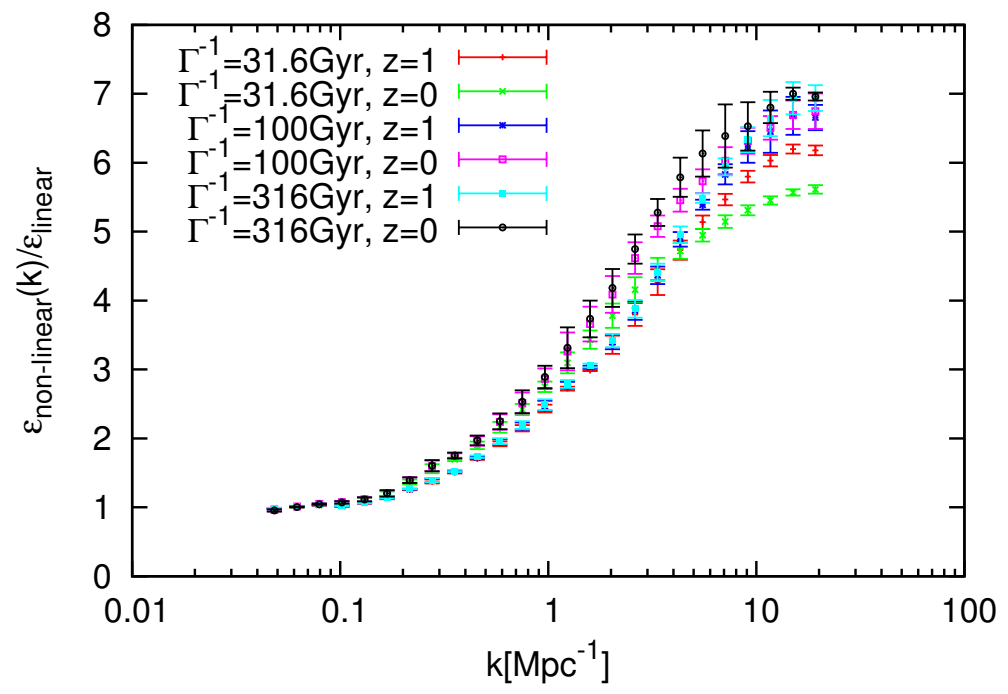

Figure 5. Enhancement of suppression from linear to non-linear matter power spectrum $\epsilon_{\text {non-linear }}(k) / \epsilon_{\text {linear }}$ for $\Gamma^{-1}=31.6,100,316 \mathrm{Gyr}$ and $z=0,1$. Here we adopt the result of N-body simulations with a box size $L=200 h_{\emptyset}^{-1} \mathrm{Mpc}$.

where $a, b, p$ and $q$ are functions of $\Gamma$ and $z$ as

$$
\begin{aligned}
& a(\Gamma, z)=0.7208+2.027\left(\frac{\Gamma}{\mathrm{Gyr}^{-1}}\right)+3.431\left(\frac{1}{1+z}\right), \\
& b(\Gamma, z)=0.0120+2.786\left(\frac{\Gamma}{\mathrm{Gyr}^{-1}}\right)+0.6499\left(\frac{1}{1+z}\right), \\
& p(\Gamma, z)=1.045+1.225\left(\frac{\Gamma}{\mathrm{Gyr}^{-1}}\right)+0.2207\left(\frac{1}{1+z}\right), \\
& q(\Gamma, z)=0.9922+1.735\left(\frac{\Gamma}{\mathrm{Gyr}^{-1}}\right)+0.2154\left(\frac{1}{1+z}\right) .
\end{aligned}
$$

The accuracy of this fitting formula is better than $10 \%$ for $\Gamma^{-1} \geq 31$ Gyr and $z \leq 1$. We note that this fitting formula is calibrated with simulations only for the fiducial cosmological parameters in Eq. (2.6). However, having observed the fact that $\epsilon_{\text {non-linear }}(k) / \epsilon_{\text {linear }}$ varies only weakly for various $\Gamma^{-1}$ and $z$, we speculate that this quantity does not vary much for different cosmological parameters, as long as the linear power spectrum does not deviate significantly from the fiducial one. This condition is effectively satisfied when one compares model predictions with observed data of matter power spectrum or CMB.

\section{References}

[1] P. A. R. Ade et al. [Planck Collaboration], arXiv:1502.01589 [astro-ph.CO].

[2] P. A. R. Ade et al. [Planck Collaboration], Astron. Astrophys. 571, A16 (2014) [arXiv:1303.5076 [astro-ph.CO]].

[3] J. Hamann and J. Hasenkamp, JCAP 1310, 044 (2013) [arXiv:1308.3255 [astro-ph.CO]]. 
[4] R. A. Battye and A. Moss, Phys. Rev. Lett. 112, no. 5, 051303 (2014) [arXiv:1308.5870 [astro-ph.CO]].

[5] A. Petri, J. Liu, Z. Haiman, M. May, L. Hui and J. M. Kratochvil, arXiv:1503.06214 [astro-ph.CO].

[6] F. Beutler et al. [BOSS Collaboration], Mon. Not. Roy. Astron. Soc. 444, 3501 (2014) [arXiv:1403.4599 [astro-ph.CO]].

[7] N. MacCrann, J. Zuntz, S. Bridle, B. Jain and M. R. Becker, arXiv:1408.4742 [astro-ph.CO].

[8] R. A. Battye, T. Charnock and A. Moss, arXiv:1409.2769 [astro-ph.CO].

[9] M. A. Buen-Abad, G. Marques-Tavares and M. Schmaltz, arXiv:1505.03542 [hep-ph].

[10] K. Osato, M. Shirasaki and N. Yoshida, arXiv:1501.02055 [astro-ph.CO].

[11] S. Ando and K. Ishiwata, arXiv:1502.02007 [astro-ph.CO].

[12] R. Flores, G. R. Blumenthal, A. Dekel and J. R. Primack, Nature 323, 781 (1986).

[13] K. Takahashi, M. Oguri and K. Ichiki, Mon. Not. Roy. Astron. Soc. 352, 311 (2004) [astro-ph/0312358].

[14] K. Ichiki, M. Oguri and K. Takahashi, Phys. Rev. Lett. 93, 071302 (2004) [astro-ph/0403164].

[15] M. Y. Wang and A. R. Zentner, Phys. Rev. D 82, 123507 (2010) [arXiv:1011.2774 [astro-ph.CO]].

[16] S. Aoyama, K. Ichiki, D. Nitta and N. Sugiyama, JCAP 1109, 025 (2011) [arXiv:1106.1984 [astro-ph.CO]].

[17] S. Aoyama, T. Sekiguchi, K. Ichiki and N. Sugiyama, JCAP 1407, 021 (2014) [arXiv:1402.2972 [astro-ph.CO]].

[18] B. Audren, J. Lesgourgues, G. Mangano, P. D. Serpico and T. Tram, arXiv:1407.2418 [astro-ph.CO].

[19] É. Aubourg, S. Bailey, J. E. Bautista, F. Beutler, V. Bhardwaj, D. Bizyaev, M. Blanton and M. Blomqvist et al., arXiv:1411.1074 [astro-ph.CO].

[20] R. Cen, Astrophys. J. 546, L77 (2001) [astro-ph/0005206].

[21] M. Oguri, K. Takahashi, H. Ohno and K. Kotake, Astrophys. J. 597, 645 (2003) [astro-ph/0306020].

[22] A. H. G. Peter, Phys. Rev. D 81, 083511 (2010) [arXiv:1001.3870 [astro-ph.CO]].

[23] V. Springel, N. Yoshida and S. D. M. White, New Astron. 6, 79 (2001) [astro-ph/0003162].

[24] V. Springel, Mon. Not. Roy. Astron. Soc. 364, 1105 (2005) [astro-ph/0505010].

[25] A. H. G. Peter, C. E. Moody and M. Kamionkowski, Phys. Rev. D 81, 103501 (2010) [arXiv:1003.0419 [astro-ph.CO]].

[26] M. Y. Wang, R. A. C. Croft, A. H. G. Peter, A. R. Zentner and C. W. Purcell, Phys. Rev. D 88, no. 12, 123515 (2013) [arXiv:1309.7354 [astro-ph.CO]].

[27] D. Cheng, M.-C. Chu and J. Tang, arXiv:1503.05682 [astro-ph.CO].

[28] Y. Suto, Astrophys. J. 321, 36 (1987).

[29] M. Y. Wang, A. H. G. Peter, L. E. Strigari, A. R. Zentner, B. Arant, S. Garrison-Kimmel and M. Rocha, Mon. Not. Roy. Astron. Soc. 445, 614 (2014) [arXiv:1406.0527 [astro-ph.CO]].

[30] C. Heymans, E. Grocutt, A. Heavens, M. Kilbinger, T. D. Kitching, F. Simpson, J. Benjamin and T. Erben et al., Mon. Not. Roy. Astron. Soc. 432, 2433 (2013) [arXiv:1303.1808 [astro-ph.CO]]. 
[31] Z. Berezhiani, A. D. Dolgov and I. I. Tkachev, arXiv:1505.03644 [astro-ph.CO].

[32] C. P. Ma and E. Bertschinger, Astrophys. J. 455, 7 (1995) [astro-ph/9506072].

[33] A. Lewis, A. Challinor and A. Lasenby, Astrophys. J. 538, 473 (2000) [astro-ph/9911177].

[34] D. Blas, J. Lesgourgues and T. Tram, JCAP 1107, 034 (2011) [arXiv:1104.2933 [astro-ph.CO]].

[35] M. Crocce, S. Pueblas and R. Scoccimarro, Mon. Not. Roy. Astron. Soc. 373, 369 (2006) [astro-ph/0606505].

[36] A. Lewis and S. Bridle, Phys. Rev. D 66, 103511 (2002) [astro-ph/0205436].

[37] M. Bartelmann and P. Schneider, Phys. Rept. 340, 291 (2001) [astro-ph/9912508].

[38] B. L'Huillier, Astrophysics Source Code Library, 1403.015 (2014) [http://adsabs.harvard.edu/abs/2014ascl.soft03015L].

[39] P. A. R. Ade et al. [Planck Collaboration], arXiv:1303.5075 [astro-ph.CO].

[40] C. L. Bennett et al. [WMAP Collaboration], Astrophys. J. Suppl. 208, 20 (2013) [arXiv:1212.5225 [astro-ph.CO]].

[41] F. Beutler, C. Blake, M. Colless, D. H. Jones, L. Staveley-Smith, L. Campbell, Q. Parker and W. Saunders et al., Mon. Not. Roy. Astron. Soc. 416 (2011) 3017 [arXiv:1106.3366 [astro-ph.CO]].

[42] L. Anderson, E. Aubourg, S. Bailey, D. Bizyaev, M. Blanton, A. S. Bolton, J. Brinkmann and J. R. Brownstein et al., Mon. Not. Roy. Astron. Soc. 427, no. 4, 3435 (2013) [arXiv:1203.6594 [astro-ph.CO]].

[43] N. Padmanabhan, X. Xu, D. J. Eisenstein, R. Scalzo, A. J. Cuesta, K. T. Mehta and E. Kazin, Mon. Not. Roy. Astron. Soc. 427, no. 3, 2132 (2012) [arXiv:1202.0090 [astro-ph.CO]].

[44] P. A. R. Ade et al. [Planck Collaboration], arXiv:1303.5077 [astro-ph.CO].

[45] R. Takahashi, M. Sato, T. Nishimichi, A. Taruya and M. Oguri, Astrophys. J. 761, 152 (2012) [arXiv:1208.2701 [astro-ph.CO]].

[46] S. Miyazaki et al., Proc. SPIE, 6269, 9 (2006).

[47] T. Abbott et al. [Dark Energy Survey Collaboration], astro-ph/0510346.

[48] R. Laureijs et al. [EUCLID Collaboration], arXiv:1110.3193 [astro-ph.CO]. 